\title{
The molecular framework of pediatric-type diffuse gliomas: shifting toward the revision of the WHO classification of tumors of the central nervous system
}

\author{
Takashi Komori $^{1}$ \\ Published online: 5 January 2021 \\ (c) The Japan Society of Brain Tumor Pathology 2021
}

While the classifications of adult diffuse gliomas according to the WHO classification of tumors of the central nervous system (CNS) were considerably updated in the 2016 revision (WHO2016CNS) based on the isocitrate dehydrogenase (IDH) mutation and $1 \mathrm{p} / 19 \mathrm{q}$ codeletion $[6,10]$, the classification of diffuse gliomas in children and adolescents has not been updated due to a lack of sufficient evidence to do so. The WHO2016CNS instead only gave these lesions tentative nomenclature, such as "pediatric diffuse astrocytoma" and "pediatric-type oligodendroglioma" as a boxed article [10].

The histopathological features of pediatric-type diffuse gliomas (pDGs) are generally non-specific, showing a wide range of variety with significant overlap. They also focally resemble circumscribed gliomas, such as pilocytic astrocytoma [1,13] and glioneuronal tumors [7]. It is often difficult to determine whether a lesion is diffuse or circumscribed based on a small specimen [1]. The histological features also overlap with those of adult diffuse glioma, which requires conventional glioma regimens combined with chemoradiotherapy that may worsen the normal development of children [8]. Genetic alterations associated with pDG also show significant overlap not only within pDG but also across all gliomas. For instance, the BRAF V600E mutation, which is the most prevalent in pDG, may be present in adult malignant gliomas, such as epithelioid glioblastoma [10]. In addition to these difficulties, pDG may occur in adults, creating further confusion over the diagnosis and treatment of adult gliomas (Fig. 1).

Takashi Komori

komori-tk@igakuken.or.jp

1 Department of Laboratory Medicine and Pathology (Neuropathology), Tokyo Metropolitan Neurological Hospital, Tokyo, Japan
To address such issues, the Consortium to Inform Molecular and Practical Approaches to CNS Tumor Taxonomy (cIMPACT-NOW), which will presumably guide future CNS tumor classification, has proposed a novel molecularly oriented approach to the typing of pDG in its updates 4 [2] and 6 [11] (Table 1).

Compared to their adult counterpart, pDGs generally have a prolonged clinical course, even with incomplete resection [16, 18], and may cause long histories (often 2 years or more) of drug-resistant epilepsy; such tumors are thus referred to as long-term epilepsy-associated tumors (LEATs) [17]. pDG often lacks genetic alterations common in adult-type gliomas, such as IDH mutations and $1 \mathrm{p} / 19 \mathrm{q}$ codeletion, and harbor distinctive genetic features [14]. For example, low-grade lesions mainly in the cerebral hemisphere often induce alterations in the RAS/MAPK pathway with a single genetic event [12, 20]. In contrast, the malignant ones, occurring in the midline structures, possess somatic mutations in genes encoding histone $\mathrm{H} 3$ isoforms, resulting in $\mathrm{K} 27 \mathrm{M}$ mutations [5]. Some cerebral hemispheric high-grade gliomas may be associated with histone $\mathrm{H} 3 \mathrm{G} 34 \mathrm{~V} / \mathrm{R}$ mutations $[3,19]$. A unique example is a high-grade cerebral astrocytoma in infant that harbors receptor tyrosine kinase (RTK) fusions, including those in the NTRK family [9, 20]. This tumor often develops as a large, cystic, circumscribed mass with neuronal differentiation and generally has a higher survival rate than seen in typical high-grade gliomas. Other diffuse cerebral high-grade gliomas with glioblastoma-like histology without IDH and H3 mutations can be grouped under the umbrella designation "diffuse pediatric-type high-grade gliomas." Figure 2 shows the major histology and genetic alterations of pDG $[4,15]$.

In summary, pDGs need to be distinguished from their adult-type counterparts as their biological behavior and genetic background are significantly different. 
Fig. 1 A schematic framework of pediatric- and adult-types diffuse gliomas. The orange triangles represent high-grade tumors, while the blue one represents low-grade tumors. Note that the pediatric type may occur in adults, and vice versa. IDH isocitrate dehydrogenase, Histone $\mathrm{H} 3$ histone $\mathrm{H} 3$ mutation, RAS/MAPK RAS, and MAP kinase pathway

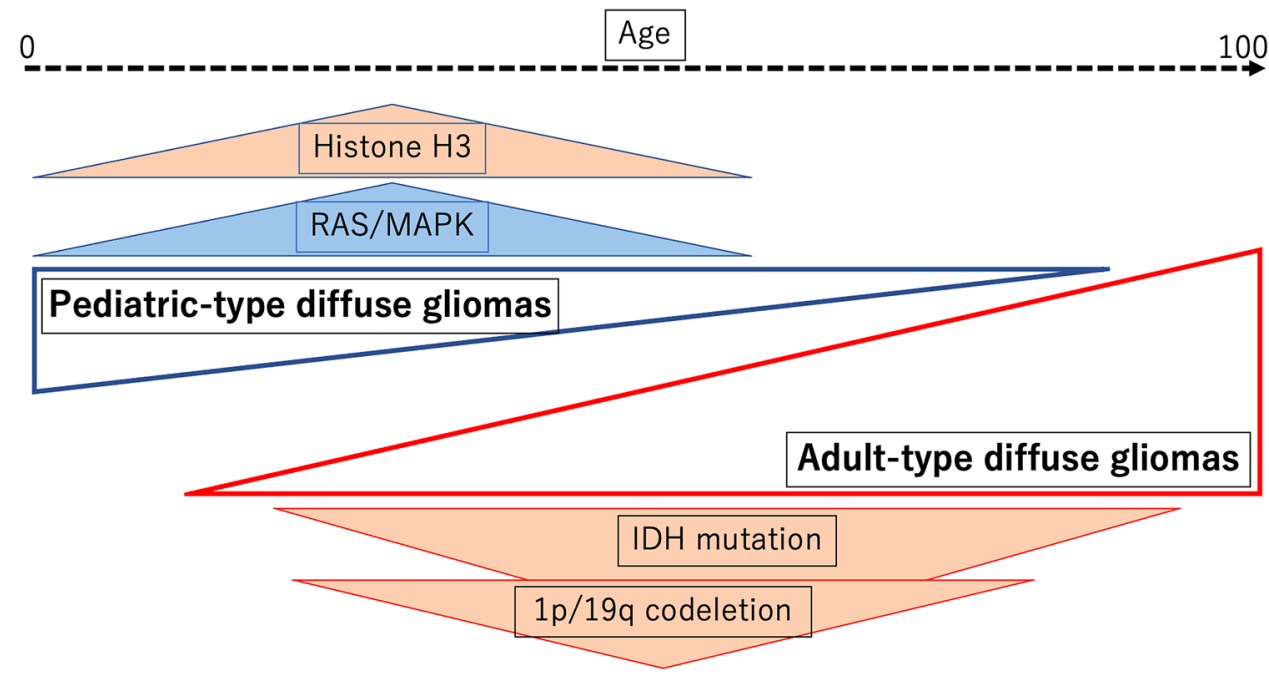

Table 1 Outline of gliomas, glioneuronal and neuronal tumors

1. Adult-type diffuse gliomas

Astrocytoma, IDH-mutant

Oligodendroglioma, IDH-mutant and $1 \mathrm{p} / 19 \mathrm{q}$ codeleted

Glioblastoma, IDH-wild type

2. Pediatric-type diffuse gliomas

Pediatric-type low-grade gliomas

Pediatric-type high-grade gliomas

3. Circumscribed astrocytomas

Pilocytic astrocytoma

Others

4. Glioneuronal and neuronal tumors

Ganglioglioma

Others

5. Ependymal tumors
Unfortunately, a purely molecular approach is currently not always available in daily diagnostic practice, particularly in resource-limited settings. However, such an approach would be scientifically rational and certainly aid in ensuring an accurate diagnosis when only a small specimen is available. Although none of the genetic alterations associated with pDG are histologically or anatomically specific, each alteration's prevalence conspicuously depends on the tumor's histology and location. As such, sequential testing of genetic alterations based on the layered integrated approach while considering the histology, location, and genetic information is highly recommended.
Fig. 2 Major histology and genetic alterations in pediatrictype diffuse gliomas. Tumor types shown in the larger font are the most common. Those in the smaller font are rare. H3 histone H3, FGFR1-TKD FGFR1-TKD fusion, $M Y B-Q K 1$ MYB-QK1 fusion, MAPK MAP kinase pathway, $w t$ wild type, and others represent mutations and alterations. For further details, see Jones, DTW [4] and Ryall, S [15]

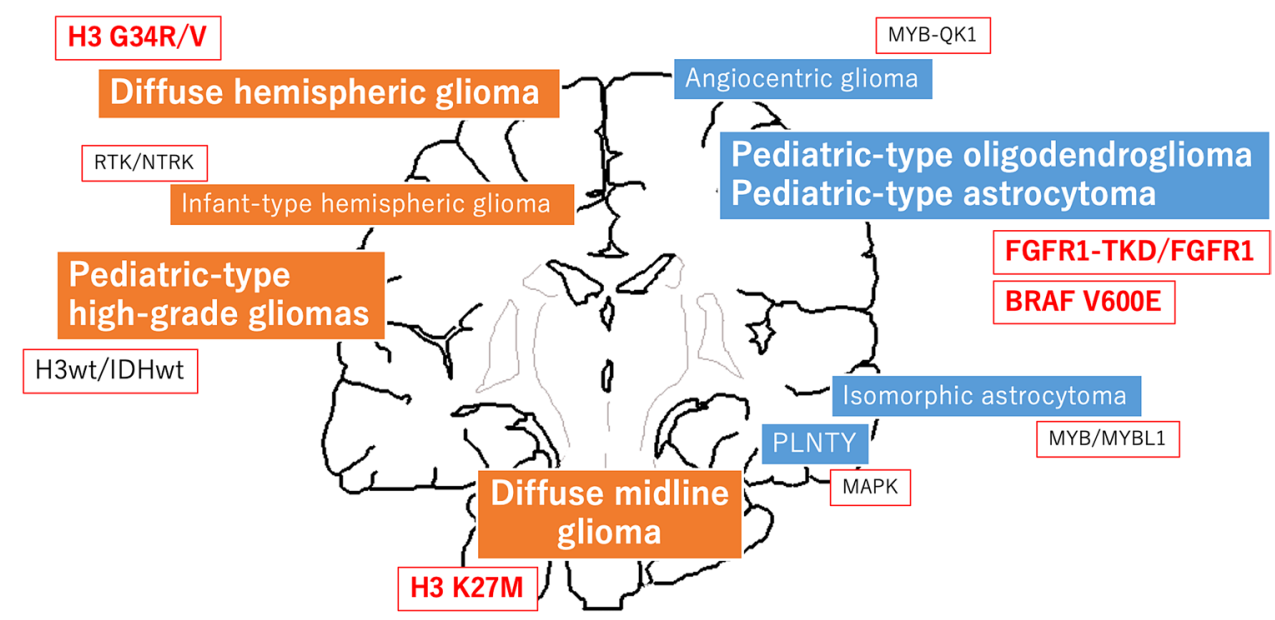

High-grade 


\section{References}

1. Collins VP, Jones DTW, Giannini C (2015) Pilocytic astrocytoma: pathology, molecular mechanisms and markers. Acta Neuropathol 129:775-788

2. Ellison DW, Hawkins C, Jones DTW et al (2019) cIMPACT-NOW update 4: diffuse gliomas characterized by MYB, MYBL1, or FGFR1 alterations or BRAF(V600E) mutation. Acta Neuropathol 137:683-687

3. Jain SU, Khazaei S, Marchione DM et al (2020) Histone H3.3 G34 mutations promote aberrant PRC2 activity and drive tumor progression. Proc Natl Acad Sci USA 117:27354-27364

4. Jones DTW, Bandopadhayay P, Jabado N (2019) The power of human cancer genetics as revealed by low-grade gliomas. Annu Rev Genet 53:483-503

5. Khuong-Quang DA, Buczkowicz P, Rakopoulos P et al (2012) K27M mutation in histone H3.3 defines clinically and biologically distinct subgroups of pediatric diffuse intrinsic pontine gliomas. Acta Neuropathol 124:439-447

6. Komori $\mathrm{T}$ (2020) Updating the grading criteria for adult diffuse gliomas: beyond the WHO2016CNS classification. Brain Tumor Pathol 37:1-4

7. Komori T, Arai N (2013) Dysembryoplastic neuroepithelial tumor, a pure glial tumor? Immunohistochemical and morphometric studies. Neuropathology 33:459-468

8. Krishnatry R, Zhukova N, Guerreiro Stucklin AS et al (2016) Clinical and treatment factors determining long-term outcomes for adult survivors of childhood low-grade glioma: a populationbased study. Cancer 122:1261-1269

9. Kurozumi K, Nakano Y, Ishida J et al (2019) High-grade glioneuronal tumor with an ARHGEF2-NTRK1 fusion gene. Brain Tumor Pathol 36:121-128

10. Louis DN, Ohgaki H, Wiestler OD et al (2016) WHO classification of tumours of the central nervous system. International Agency for Research On Cancer, Lyon

11. Louis DN, Wesseling P, Aldape K et al (2020) cIMPACT-NOW update 6: new entity and diagnostic principal recommendations of the cIMPACT-Utrecht meeting on future CNS tumor classification and grading. Brain Pathol. https://doi.org/10.1111/bpa.12832

12. Northcott PA, Pfister SM, Jones DT (2015) Next-generation (epi) genetic drivers of childhood brain tumours and the outlook for targeted therapies. Lancet Oncol 16:e293-302

13. Pujadas E, Chen L, Rodriguez FJ (2019) Pathologic and molecular aspects of anaplasia in circumscribed gliomas and glioneuronal tumors. Brain Tumor Pathol 36:40-51

14. Reuss DE, Mamatjan Y, Schrimpf D et al (2015) IDH mutant diffuse and anaplastic astrocytomas have similar age at presentation and little difference in survival: a grading problem for WHO. Acta Neuropathol 129:867-873

15. Ryall S, Tabori U, Hawkins C (2020) Pediatric low-grade glioma in the era of molecular diagnostics. Acta Neuropathol Commun $8: 30$

16. Tateishi K, Nakamura T, Yamamoto T (2019) Molecular genetics and therapeutic targets of pediatric low-grade gliomas. Brain Tumor Pathol 36:74-83

17. Thom M, Blumcke I, Aronica E (2012) Long-term epilepsy-associated tumors. Brain Pathol 22:350-379

18. Wisoff JH, Sanford RA, Heier LA et al (2011) Primary neurosurgery for pediatric low-grade gliomas: a prospective multi-institutional study from the Children's Oncology Group. Neurosurgery 68:1548-1554 (discussion 1554-1545)

19. Yoshimoto K, Hatae R, Sangatsuda Y et al (2017) Prevalence and clinicopathological features of H3.3 G34-mutant high-grade gliomas: a retrospective study of 411 consecutive glioma cases in a single institution. Brain Tumor Pathol 34:103-112

20. Zhang J, Wu G, Miller CP et al (2013) Whole-genome sequencing identifies genetic alterations in pediatric low-grade gliomas. Nat Genet 45:602-612

Publisher's Note Springer Nature remains neutral with regard to jurisdictional claims in published maps and institutional affiliations. 